\title{
Fabrication and characterization of cross-linked gelatin electro-spun nano-fibers
}

\author{
Thi-Hiep Nguyen, Byong-Taek Lee
}

Department of Biomedical Engineering and Materials, College of Medicine, Soonchunhyang University, Cheonan, Korea Email: $\underline{\text { lbt@sch.ac.kr }}$

Received 5 Octorber 2010; revised 15 Octorber 2010; accepted 18 Octorber 2010.

\begin{abstract}
In this study, we developed a fast, simple and novel process to fabricate cross-linked electro-spun gelatin with limited amounts of glutaraldehyde (GA) using trifluoroacetic acid (TFA) as the solvent. Using SEM, the uncross-linked gelatin fibers were determined to have diameters between $50-300 \mathrm{~nm}$, while the cross-linked gelatin electro-spun fibers had diameters between 100-500 $\mathrm{nm}$. FT-IR revealed that the uncross-linked and cross-linked electro-spun gelatin was fabricated successfully by electro-spinning using TFA as a solvent, which has not been reported until now. Stress-strain curves showed that the addition of small amounts of GA increased the strength of the gelatin by two fold and allowed for the creation of a water insoluble gelatin electro-spun membrane.
\end{abstract}

Keywords: Gelatin, Cross-Linking, Glutaraldehyde

\section{INTRODUCTION}

Collagen is an important protein for cell proliferation and is found in the extracellular matrix (ECM). M.D. Johnson reported that collagen is one of the main fibrous elements in connective tissue [1]. Gelatin is a natural biopolymer that is prepared by partial hydrolysis of collagens. Most of these structural proteins are found in the skin, tendons, cartilage, bones and connective tissues of animals. Gelatin (type A, obtained from porcine skin, $\sim 300$ Bloom) prepared from acid-treated collagens has a biocompatibility that is similar to collagen but is much cheaper to produce. Therefore, gelatin is extensively used in medical products, such as wound dressings [2], drug delivery systems [3], nerves [4], etc. Gelatin has been widely applied because it has three excellent features; first, it has a high biocompatibility, biodegradability and bioactivity [5]. Second, it can improve water absorption of non-absorptive polymers such as poly- caprolactone [6], poly (lactic-co-glycolic acid) (PLGA) [4], etc. Finally, gelatin can react with - $\mathrm{OH}$ groups and positively charged polymers such as polyvinyl alcohol (PVA) [7], chitosan [8] to create 3-D porous sponges where gelatin is involved in physical and/or chemical reactions.

Electro-spinning has recently been shown to be a useful method to generate fibrous scaffolds for tissue engineering applications and has been used in a broad range of different research fields [9]. Electro-spinning is an inexpensive, effective, and simple method to produce non-woven nano-fibrous mats, which have intrinsically high surface to volume ratios, increased flexibility in surface functionalities, improved mechanical performances, and smaller pores than fibers produced using traditional methods [10]. The advantages of the electro-spinning technique include the production of very thin fibers that are on the order of a few nanometers or micrometers with large surface areas, ease of functionalization for various applications, superior mechanical properties and ease of processing [11]. Decreasing the fiber diameter within these mats causes many beneficial effects, including increased specific surface area to volume ratios. Electro-spun scaffolds allow cells to grow while providing sufficient mechanical support. Therefore, various biodegradable as well as synthetic polymers [12], peptide copolymers [13], and natural proteins [14] have been electro-spun into micro/nano-fibers for a multitude of biomedical applications such as scaffolds for use in tissue engineering [15], wound dressing [16], drug delivery [17], and vascular grafts [18]. The necessary components of an electro-spinning apparatus include a high power voltage supply, a capillary tube with a needle or pipette, and a collector that typically consists of a conducting material. [19] The solvent is the most important factor in the electro-spinning operation. In previous studies, electro-spun gelatin was fabricated using various solvents such as 2,2,2-triflourothanol (TFE) [20], wa- 
ter/water in acetic acid or mixed with ethyl acetate [21]. However, Trifluoroacetic acid (TFA) has not yet been used as a solvent for the fabrication of electro-spun gelatin even though collagen [22] and chitosan [10] have been fabricated using TFA as a co-solvent.

When the inherently beneficial effects of gelatin material are combined with the enhanced properties of nano-fibrous mats, applications arise in a wide range of tissue engineering fields. In this work, we developed a novel one-step process to fabricate cross-linked electro-spun gelatin nano-fibers that was faster and more economical than the previously reported two-step vapor-cross-linking method [20]. In this study, the electro-spun gelatin was fabricated using TFA as a solvent and cross-linked electro-spun gelatin was produced by adding small amounts of glutaraldehyde (GA) to the gelatin solution. Small amounts of GA were shown to be nontoxic and increased the mechanical strength by two-fold, relative to the uncross-linked gelatin electro-spun mats [3,5,23-25]. SEM morphology, DSC and FT-IR demonstrated that uncross-linked and cross-linked electro-spun gelatin was successfully fabricated by electro-spinning using TFA as a solvent. In the in vitro biodegradable studies, the morphology of the gelatin mat was found to change due to swelling and biodegradability. Thus, the cross-linked electro-spun gelatin holds promise for use in tissue engineering and biomedical applications.

\section{MATERIALS AND METHODS}

\subsection{Materials}

Gelatin (from porcine skin, type A) was purchased from Sigma-Aldrich USA. Trifluoroacetic acid (TFA, CF3 $\mathrm{COOH}, 99.0 \%)$ was purchased from Duksan Pure Chemical Co., Korea. Glutaraldehyde (GA) was obtained from DeaJung Co., Korea. Fetal bovine serum (FBS), P.S. (penicillin/streptomycin (antibiotics)), Dulbecco's phosphate buffered saline (D-PBS) without calcium or magnesium, MTT solution and trypsin-EDTA were purchased from GIBCO (Carlsbad, CA). The L-929 cell line was obtained from the ATCC Cell Line (CCL-1TM, NCTC clone 929 [L cell, L-929, derivative of Strain L], Korea). DMSO (Dimethylsulfoxide 99, 0\%) was purchased from, Samchun Pure Chemical Co., LTD (Korea).

\subsection{Preparation of Polymer Solutions}

A 15 wt \% gelatin solution was prepared by adding a TFA solution to $1.5 \mathrm{~g}$ of gelatin until the solution weighed $10 \mathrm{~g}$. The gelatin was completely dissolved by stirring for 6 hours. $1.5 \mathrm{ml}$ of $0.05 \% \mathrm{wt} / \mathrm{v}$ GA in PBS was then added to $10 \mathrm{ml}$ of the $15 \mathrm{wt} \%$ gelatin.

\subsection{Electro-spinning Setting}

The gelatin solutions were placed in a plastic syringe (lure-lock type, $12 \mathrm{ml}$ ) that was fitted to a needle with a 25 gauge (inner diameter $0.25 \mathrm{~mm}$ ) tip diameter. The flow rate of the CS solutions $(0.5 \mathrm{ml} / \mathrm{h})$ was controlled using a syringe pump (lure-lock type, Korea). The electro-spinning voltage $(10 \mathrm{kV})$ was supplied directly by a high DC voltage power supply (NNC-30 kilovolts-2mA portable type, Korea). A grounded steel plate located 15 centimeters away from the tip of the syringe needle was used to collect the nano-fiber mats. The structure of the gelatin fibrous mats fabricated with TFA as a solvent was observed by SEM (SM-65F, JEOL, and Japan).

\subsection{Characterizations}

\subsubsection{Fourier Transform-Infrared Spectra (FT-IR)}

The cross-linked gelatin was characterized by attenuated reflectance Fourier transform spectroscopy (Spectrum GX, PerkinElmer, USA). The infrared spectra of the samples were measured over a wavelength range of $4000-500 \mathrm{~cm}^{-1}$. All spectra were acquired in the spectral range through the accumulation of 64 scans with a resolution of $4 \mathrm{~cm}^{-1}$.

\subsubsection{Tensile Strength}

Tensile strength of all samples was tested using the R\&B Model Unitech Series (Universal Testing Machine). The dimensions of the samples (width $2 \mathrm{~mm}$, length $27 \mathrm{~mm}$ ) were measured using a digital micrometer and the thickness $(100 \mu \mathrm{m})$ was measured by SEM prior to the measurement of tensile strength. Sample preparation and measurement of the tensile strength have been described in our previous reports [26].

\subsubsection{Differential Scanning Calorimetry (DSC)} DSC measurements (METTLER TOLEDO KOREA DSC822e) were conducted using a sample weight of 3-5 mg under a nitrogen atmosphere and at a scanning speed of $10^{\circ} \mathrm{C} / \mathrm{min}$. The samples were heated from 0 to $250^{\circ} \mathrm{C}$ at a rate of $10^{\circ} \mathrm{C} / \mathrm{min}$.

\subsection{Characterization of Gelatin in Vitro Study}

To characterize the in vitro properties of the gelatin mat, 9 samples for each group were cut into square shapes (1 $\mathrm{cm} \times 1 \mathrm{~cm})$ and then immersed in simulated body fluid (SBF) at $37^{\circ} \mathrm{C}$ for $30 \mathrm{~min}, 120 \mathrm{~min}, 720 \mathrm{~min}$ and 1440 min.

\subsubsection{Swelling Behavior of Gelatin Membranes}

The swelling behavior of the gelatin mat was measured by swelling the electro-spun mat in an SPF solution in a humid environment $\left(6 \% \mathrm{CO}_{2}, 37^{\circ} \mathrm{C}\right.$ in an incubator). The weight of the dry gelatin mat (approximately $0.05 \mathrm{~g}$ ) and the wet gelatin mat removed from the SPF solution at 
different times was then determined. Their wet weights were established after first blotting with a filter paper followed by blowing with a stream of air to remove surface water. The swelling ratio was calculated using the following equation:

$$
\text { Esr }(\%)=((\mathrm{Ws}-\mathrm{Wd}) / \mathrm{Wd}) \times 100
$$

(Where Esr is the water absorption (\% wt) of the membranes, and Wd and Ws are the weights of the samples in the dry and swollen states, respectively).

\subsubsection{Biodegradability of Gelatin in Vitro}

The biodegradability of the gelatin was assessed by changes in the SEM morphology after different immersion time periods.

\section{RESULTS}

\subsection{SEM Morphology of Uncross-Linked and Cross-Linked Electro-Spun Gelatin}

The nano-morphology of the uncross-linked and crosslinked electro-spun gelatin fibers is shown in Figure 1. The electro-spun gelatin mats were fabricated using an electro-spinning machine (NNC-30kV-2mA portable type, Nano NC. Korea.). The parameters used for the fabrication of each polymer fibrous mats were as follows: voltage $10 \mathrm{kV}$, distance $15 \mathrm{~cm}$, flow rate $0.5 \mathrm{ml} /$ hour and nozzle $25 \mathrm{G}$. The morphology of the gelatin nano-fibers electro-spun from gelatin in TFA without GA is shown in Figure 1a. In this case, the fiber diameter was $50-300 \mathrm{~nm}$. The random arrangement of the fibers mimicked the behavior of extra cellular matrix (ECM) proteins, which provides mechanical support and regulates cellular activities [27]. However, electro-spun gelatin is water soluble [25], which is not suitable for long-term applications. To overcome this drawback, GA was added to the gelatin solution as described above. The nano-morphology of the gelatin-GA electro-spun mat is shown in Figure 1(b) and Figure 1(c). Figure 1(b) shows the SEM morphology of the electro-spun gelatin fabricated with 15 wt \% of gelatin and $1.5 \mathrm{ml}$ of GA (0.05 wt \%) in PBS without storage in a vacuum oven. Figure 1(c) shows the SEM morphology of sample B, which was maintained in a vacuum oven at $100^{\circ} \mathrm{C}$ for 24 hours. While GA is considered a creep cross-linking agent [28], and was added to the gelatin before electro-spinning, GA could not cross-link all gelatin because of its highly acidic properties. However, when the electro-spun gelatin was stored overnight, the acid evaporated and GA was able to more extensively cross-link the gelatin. The electro-spun gelatin maintained at room temperature was referred to as cross-linked $\mathrm{A}$, while the electro-spun gelatin maintained in a vacuum oven at $100^{\circ} \mathrm{C}$ was referred to as cross-linked B. GA remained

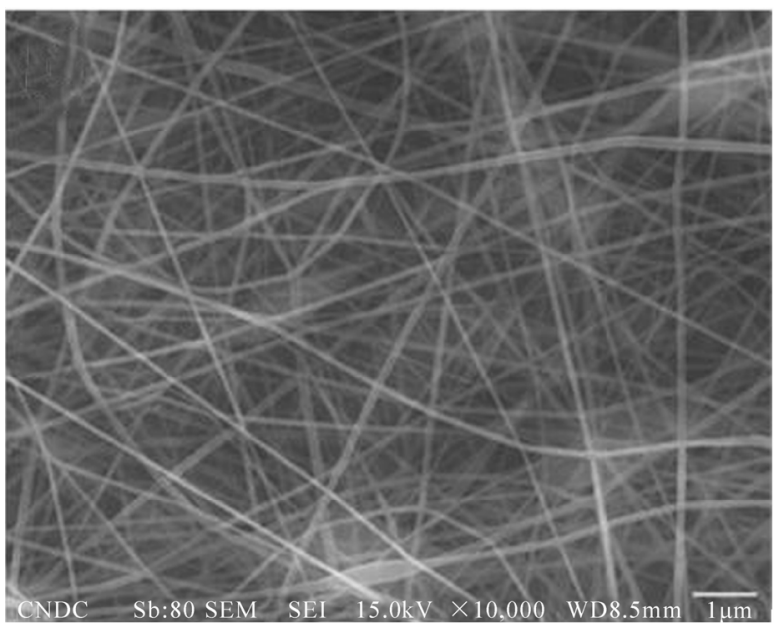

(a)

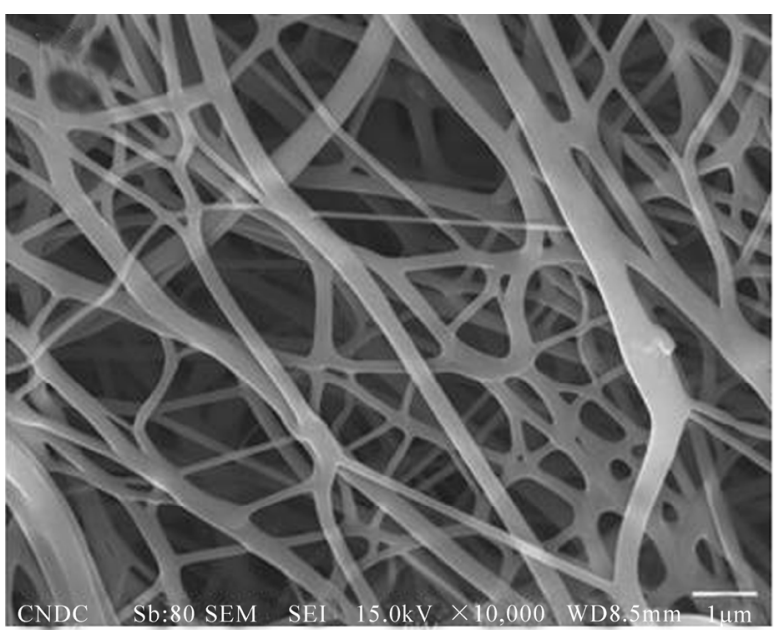

(b)

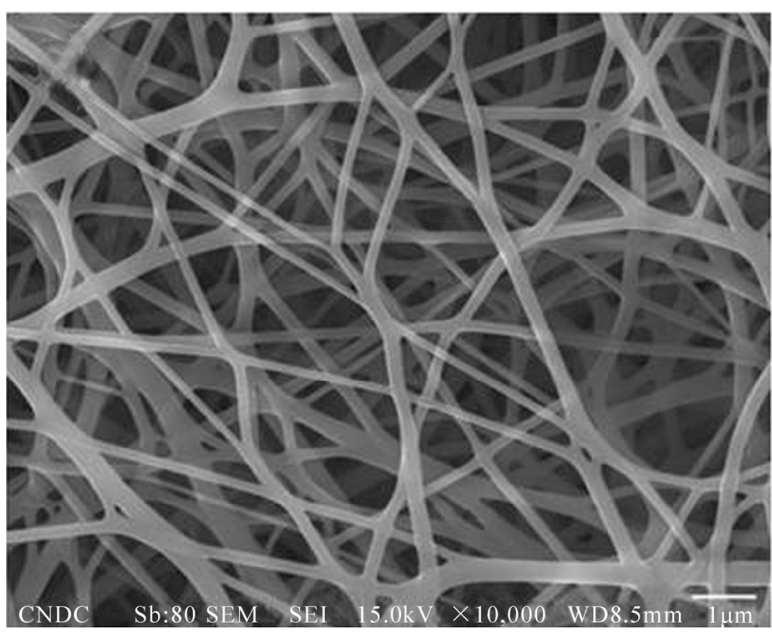

(c)

Figure 1. Uncross-linked electro-spun (a) cross-linked A gelatin electro-spun after keeping 1 day at room temperature (b) and cross-linked B gelatin after keeping 1 day in vacuum oven at $100{ }^{\circ} \mathrm{C}(\mathrm{c})$. 
on the surface of the gelatin fibers after electro-spinning and the fibers were cross-linked, as previously reported for chitosan [10]. The diameter of the electro-spun gelatin-GA was between 100-500 nm, which was larger than the un-cross-linked fibers.

\subsection{Characterization of Un-Cross-Linked and Cross-Linked Electro-Spun Gelatin Nanofibers through FTIR Analysis}

FTIR measurements were conducted on un-cross-linked gelatin and cross-linked gelatin to determine whether cross-linking of the electro-spun gelatin affected the primary gelatin structure (Figure 2). The FTIR spectrum of the un-cross-linked electro-spun gelatin revealed a structure that was similar to raw gelatin, which we described in a previous report [25]. This demonstrated that TFA did not alter the structure of raw gelatin. The un-cross-linked electro-spun gelatin had an amide I peak (C = O stretch) at1636-1640 $\mathrm{cm}^{-1}$, amide II peak (N-H bend and C-H stretch) at $1542-1544 \mathrm{~cm}^{-1}$, amide III peak (C-N stretch plus $\mathrm{N}-\mathrm{H}$ in phase bending) at $1240 \mathrm{~cm}^{-1}$ and amide A peak (N-H stretching vibration) at 3300 $\mathrm{cm}^{-1}$, which are the distinguishing features of gelatin.

The spectrum of the cross-linked gelatin is shown in greater detail in Figure 3. In addition to the previously mentioned peaks, a strong peak at $1450 \mathrm{~cm}^{-1}$ was observed in the cross-linked gelatin due to aldimine absorption [3]. In comparison to previous reports [3,5,29], we found that the amide II peak changed from smooth to several small peaks. Glutaraldehydes have an aldehyde group (-CHO) that reacts with the amino group of the lysine residues of proteins [5]. The un-cross-linked and cross-linked membranes were discriminated by a slow change in color from white to yellow. The color change occurred because the aldimine linkage $(\mathrm{CH}=\mathrm{N})$ reactions took place during the cross-linking process.

This is the first report where uncross-linked electro-spun gelatin and cross-linked electro-spun gelatin, through the addition of glutaraldehyde, were fabricating using TFA; however, TFA had been previously used as a solvent in the fabrication of electro-spun collagen [22]. The characteristic absorption of the aldimine groups occurred at $1450 \mathrm{~cm}^{-1}$. Furthermore, additional peaks were observed at $1470-1570 \mathrm{~cm}^{-1}$, which increased as the cross-linking reaction proceeded (Figure 2). To more conclusively determine if cross-linked gelatin was successfully fabricated, we expanded the FT-IR spectra from 1100-1750 $\mathrm{cm}^{-1}$ (Figure 3). In this region of the FT-IR spectra, the amount of $\mathrm{CH}=\mathrm{N}$ groups increased. Moreover, some peaks appeared in this region was similar to previous repot [5]. Thus, both un-cross-linked and cross-linked electro-spun mats gelatin were successfully fabricated via the electro-spinning method using TFA as

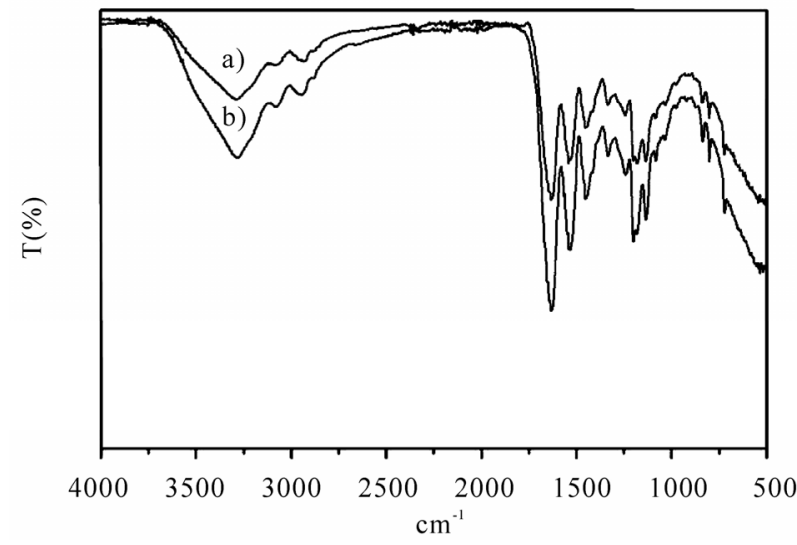

Figure 2. FT-IR of uncross-linked gelatin fibers (a) and crosslinked-B gelatin fibers (b).

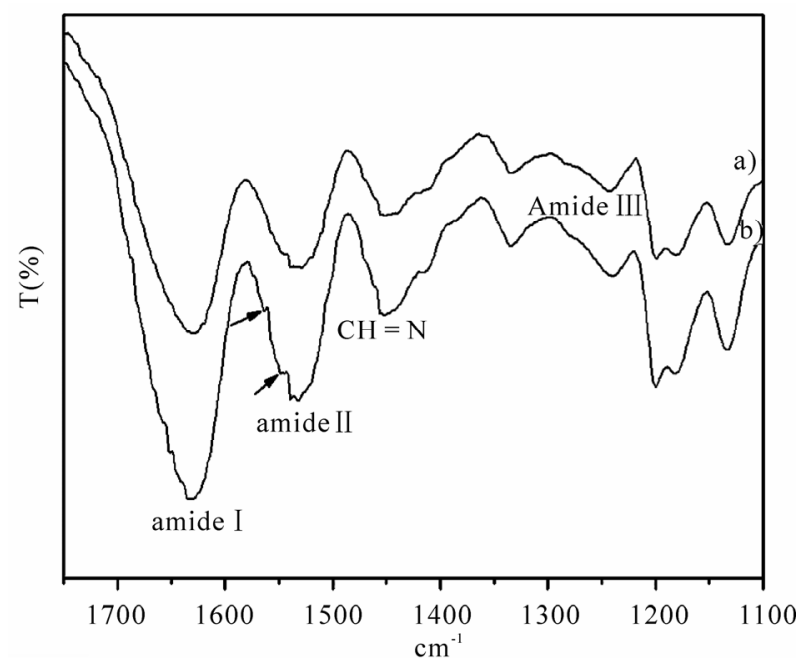

Figure 3. FT-IR of uncross-linked gelatin (a) and cross-linked$\mathrm{B}$ gelatin electrospun fibers (b).

a solvent and the differences in the spectra may have resulted from the broad background underneath each spectrum. This method allows one to control the addition of very small amounts of glutaraldehyde during the cross-linking process, which is an essential condition to limit the insolubility and increase the biocompatibility of gelatin for applications in the biomedical field [23].

\subsection{Mechanics of Uncross-Linked and Cross-Linked Gelatin Nanofiber Electrospun by Tensile Strength of Thin Membrane}

Stress-strain curves were recorded using a R\&B Model Unitech Series (Universal Testing Machine) and the cells were loaded following ASTM Standard D882-01 protocol [30] for thin membranes. For the tensile strength measurements, the samples were prepared with stripshape dimensions of $0.1 \mathrm{~mm}$ thick, $25 \mathrm{~mm}$ long and 2 
$\mathrm{mm}$ wide. The samples were then attached to the paper frame as described in reference [26]. The ratio of the stretching process was set at $0.1 \mathrm{~mm} / \mathrm{min}$.

The stress of the cross-linked electro-spun gelatin increased approximately 2 times that of the uncross-linked electro-spun gelatin (Figure 4). The uncross-linked electro-spun gelatin was fabricated using TFA as a solvent, which is more economical than 2,2,2-trifluoroethanol, and the tensile strength was similar to previous reports with a stress around $1 \mathrm{MPa}$ and a strain around $20 \%$ [20]. Moreover, the strain of the cross-linked electro-spun gelatin was $140 \%$ higher than the uncrosslinked electro-spun gelatin 5 times, which was also higher than the samples subjected to the immersion methods at the same concentration as described in ref [23]. However, the stress of the cross-linked electro-spun gelatin (2 MPa) was not significantly higher as described in ref [20]. The biocompatible properties of the crosslinked gelatin depended on the glutaraldehyde concentration, which was why only small amounts of GA were used in this study. This method is advantageous in that one can easily and reliably control the glutaraldehyde concentration, which is important for biomedical and biocompatibility applications.

Controlling the amount of GA not only decreases toxicity but also increases the biocompatibility of crosslinked electro-spun gelatin membranes and the mechanical strength, which is essential to applications involving implantation [23,24,31]. Figure 5 shows the changes in the morphology of the gelatin and the tensile fracture surfaces of uncross-linked and cross-linked electro-spun mats. Uncross-linked gelatin had brittle and abrupt break properties (Figure 5(a)). In contrast, the cross-linked gelatin had elongated and flexible proper-

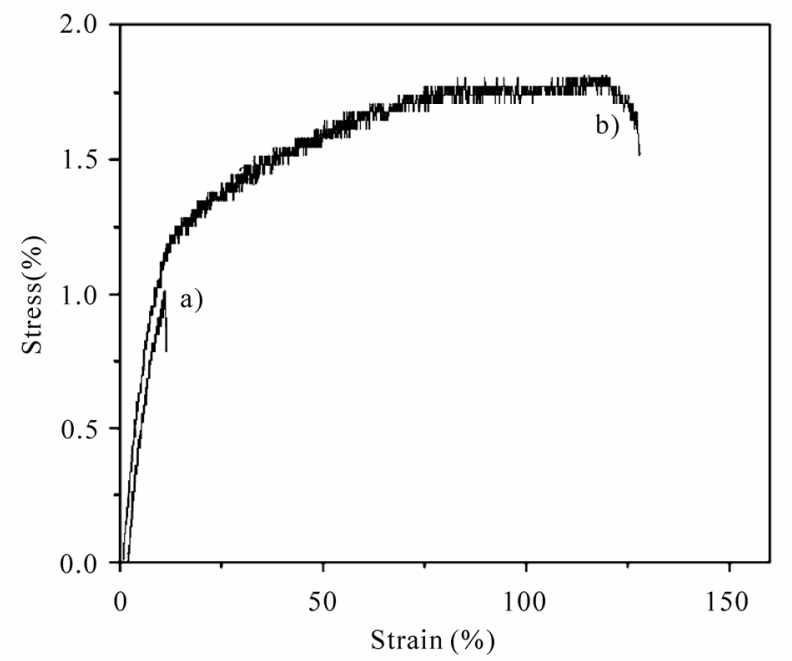

Figure 4. Tensile strength of uncross-linked gelatin (a) and cross-linked-B gelatin electro-spun fibers (b).

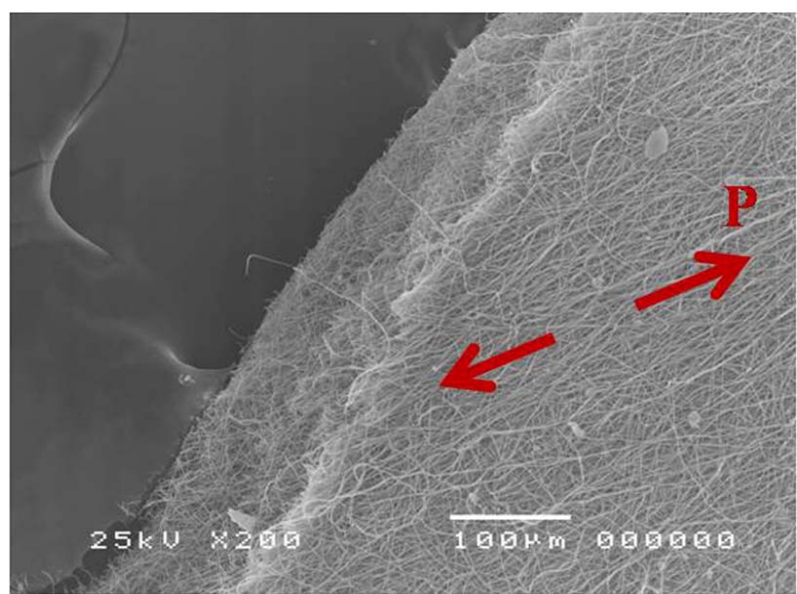

(a)

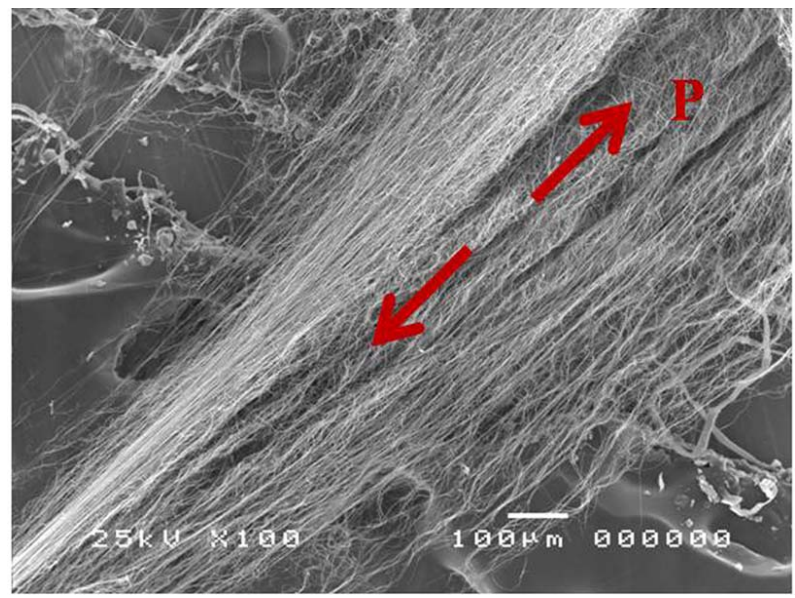

(b)

Figure 5. SEM morphology of uncross-linked gelatin (a) and cross-linked gelatin mat at the point of fracture after tensile strength (b).

ties (Figure 5(b)). Remark arrow (P) show the direction of load cells applied.

\subsection{Differential Scanning Calorimetry (DSC)}

DSC investigations are widely used to examine changes in the thermal properties of initial components and new components created after a reaction.

Figure 6 shows the DSC thermograms of gelatin powder (a), uncross-linked electro-spun gelatin mats (b) and cross linked electro spun gelatin mats (c). Two peaks were observed in the DSC scans of the gelatin powder, while only one peak was observed at approximately $95.8^{\circ} \mathrm{C}$ for the cross-linked gelatin sample, which corresponded to the endothermic peak of cross-linked gelatin nanofibers. In contrast, the endothermic peak of uncross-linked gelatin was observed at approximately $75^{\circ} \mathrm{C}$. Therefore, because of the cross-linking reaction, the $\mathrm{Tg}$ value of the uncross-linked gelatin mat changed from 


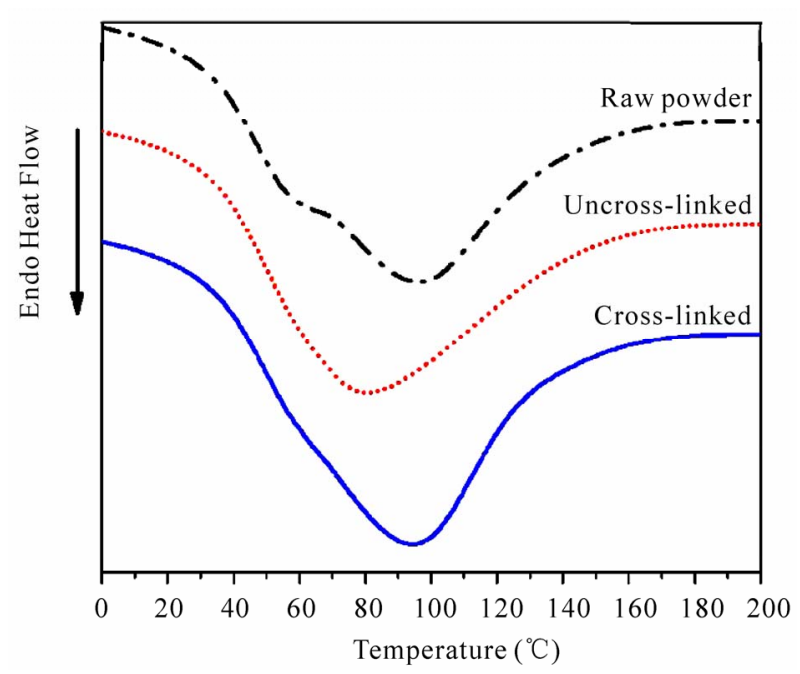

Figure 6. DSC thermograms of gelatin powder, uncross-linked electro-spun gelatin mats and cross-linked electro-spun gelatin mats.

$75^{\circ} \mathrm{C}$ to $95.8^{\circ} \mathrm{C}$.

\subsection{Characterization of Gelatin Electro-Spun in in Vitro Study}

\subsubsection{Swelling behavior of gelatin mats}

To measure the swelling ratio of uncross-linked and cross-linked electro-spun mats, the swelling ratio was determined using the procedures outlined in the Materials and Methods. As shown in Figure 7, the swelling ratio of the cross-linked electrospun gelatin mat (a) was twice that of the uncross-linked electro-spun gelatin mat (b). These results demonstrated that the swelling of the electro-spun gelatin was restricted due to the crosslinking reaction. This property is highly important for implantation applications. The ratio of both cross-linked and uncross-linked electro-spun gelatin mats demonstrated that the electro-spun mat was stable between 200-min and 1000-min.

\subsubsection{Biodegradability}

Figure 8 shows the SEM morphology of the crosslinked electro-spun mats that had been immersed in the SBF solution for varies periods of time. The SEM morphologies of the cross-linked electro-spun gelatin that had been immersed in SBF for $30 \mathrm{~min}, 120 \mathrm{~min}, 720$ min and $1440 \mathrm{~min}$ are shown in Figure 8(a), Figure 8(b), Figure 8(c) and Figure 8(d), respectively. These images show that most of the cross-linked gelatin electro-spun fibers remained intact; however, some regions of the electro-spun gelatin fibers had melted. Nevertheless, even after $1440 \mathrm{~min}$, most of the electro-spun fibers still remained intact. In the biodegradability test, no changes in biodegradability were observed when GA was added to the gelatin solution before electro-spinning.

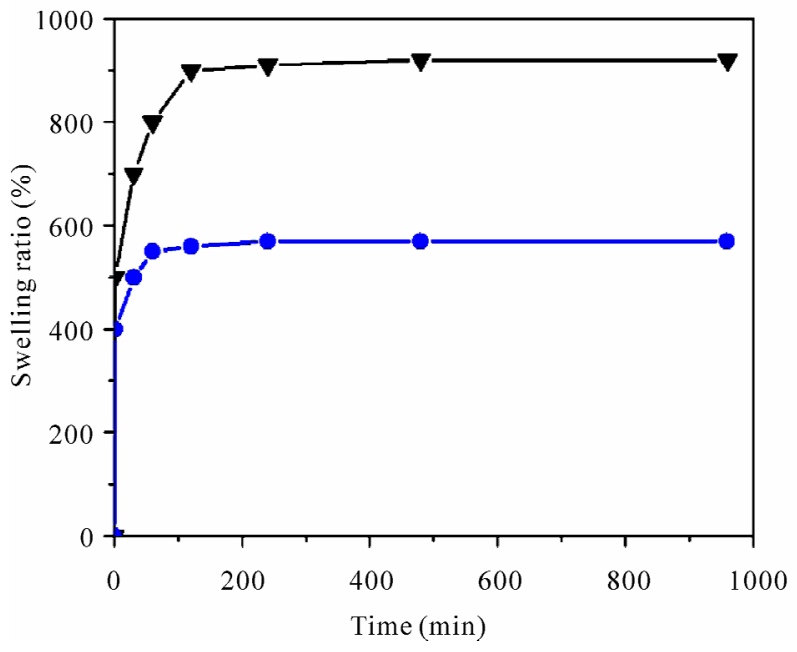

Figure 7. Swelling behavior of uncross-linked electro-spun gelatin (a) and cross-linked electro-spun gelatin (b) after immersing in the SPF solution for various periods of time.

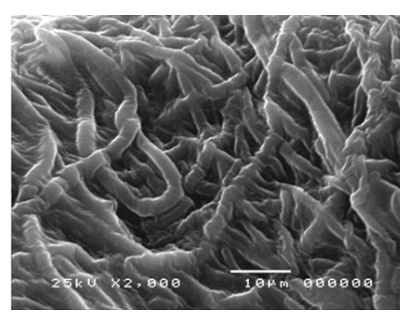

(a)

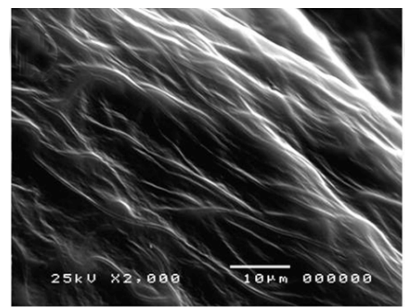

(c)

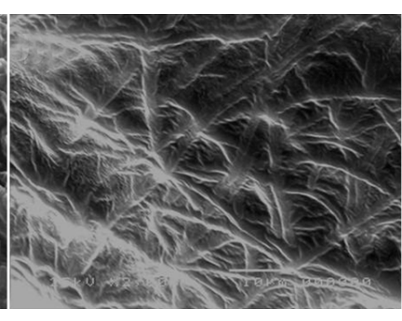

(b)

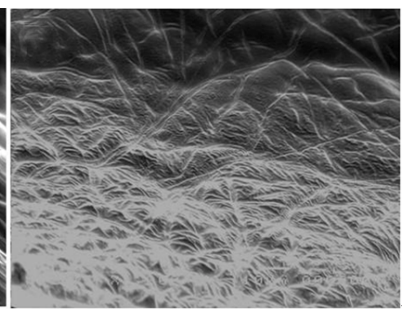

(d)
Figure 8. SEM morphology of cross-linked electro-spun gelatin after immersing in the SPF solution for $30 \mathrm{~min}$ (a), $120 \mathrm{~min}$ (b), $720 \mathrm{~min}$ (c) and $1440 \mathrm{~min}$ (d).

\section{DISCUSSION}

Gelatin derived from collagen is a highly effective fibrous matrix for cell attachment. In addition, gelatin has excellent biocompatibility, biodegradability and bioabsorption, and is widely used in biomedical, biomaterial, and tissue engineering applications. Therefore, scientists have focused on using this material as scaffolds in tissue engineering and biomedical regeneration applications. Electro-spun gelatin is similar to the collagen ECM in the human body, which is the main matrix for cell attachment and ground substance of polysaccharides, 
proteins and water [1]. However, gelatin is a water- soluble material; hence uncross-linked electro-spun gelatin is also water-soluble. To maintain the gelatin ECM, gelatin electro-spun fibrous mats should be cross-linked. In this work, glutaraldehyde was used as a cross-linking agent. However, the amount of glutaraldehyde used for cross-linking should be limited because glutaraldehyde is a toxic chemical. Previous studies cross-linked gelatin through the evaporation method. The evaporation method has two drawbacks: the first is that the cross-linking agent used to cross-link the gelatin molecule from the surface to the inside requires large amounts of GA. Second, it is difficult to control the amount of GA, which is toxic at concentrations higher than $5 \%$. To overcome these complications, we developed a novel method for fabricating cross-linking gelatin fibrous mats by adding low amounts of GA in a controlled manner to the gelatin solution, which dissolves in strong acid. This acid effect limits the cross-linking agent from cross-linking all gelatins during the electro-spinning process but this effect can be eliminated by storing at room temperature or in a vacuum oven after completion of the electro-spinning process. Finally, in this study, we obtained uncrosslinked electro-spun gelatin and cross-linked electro-spun gelatin fibers that had nanometer scale diameters. However, the diameter of the cross-linked electro-spun gelatin fibers increased from $50-300 \mathrm{~nm}$ to $100-500 \mathrm{~nm}$ compared with the uncross-linked electro-spun gelatin mats (Figure 1) Successful cross-linking of the gelatin membrane was confirmed by FT-IR, as shown in Figure 2 and Figure 3. Stress-strain curves showed that the cross-linked electro-spun gelatin membrane was stronger and more flexible. In addition, it was not brittle as we previously reported for the electro-spun cross-linked gelatin formed by the evaporated method (Figure 4). To confirm the results obtained from the stress-strain curves, surface fractures of the electro-spun gelatin were observed by SEM (Figure 5). In the DSC thermograms of uncross-linked and cross-linked electro-spun gelatin, an increase in the Tg of the cross-linked electro-spun mat was observed, demonstrating that the cross-linking agent successfully cross-linked the gelatin fiber (Figure 6). Prior to using biomaterials in implantation applications, the swelling ratio and biodegradability must be investigated. Therefore, the swelling ratios and biodegradability of electro-spun gelatin mats were investigated before the material was tested in vitro (Figure 7 and Figure 8, respectively).

\section{CONCLUSION}

In this study, we developed a novel process to fabricate cross-linked electro-spun gelatin by adding GA to the gelatin solution before electro-spinning. In this work,
TFA was used for the first time as the solvent to fabricate the electro-spun gelatin. This was also the first time that GA was added to the gelatin solution before electro-spinning to cross-link the gelatin. The FT-IR data confirmed that TFA did not alter the amide groups of the gelatin and that the mat was successfully cross-linked. The advantages of this method are: 1) the amount of GA added to the solution was easily controlled during the cross-linking process, which limits the cytotoxicity of GA activity. 2) GA can disperse onto the gelatin fibers and be cross-linked inside the fibers, which help maintain the ECM structure. 3) The small amount of added GA increased the mechanical strength of the material by two fold, and allowed for the formation of a flexible water-insoluble cross-linked gelatin electro-spun membrane.

\section{ACKNOWLEDGEMENTS}

This work was supported by the Mid-career Research Program through a NRF grant funded by MEST (NO 2009-0092808).

\section{REFERENCES}

[1] Johnson, M.D. (2000) Human Biology: Concenpts and Current Issues. San Francisco, California.

[2] Choi, Y.S., Hong, S.R., Lee, Y.M., et al. (1999). Study on gelatin-containing artificial skin: I. Preparation and characteristics of novel gelatin-alginate sponge. Biomaterials, 20, 409-417.

[3] Akin, H. and Hasircl, N. (1995) Preparation and Characterization of Crosslinked Gelatin Microspheres. Journal of Applied Polymer Science, 58, 95-100.

[4] Li, X.-K., Cai, S.-X., Liu, B., et al. (2007) Characteristics of PLGA-gelatin complex as potential artificial nerve scaffold. Colloids and Surfaces B: Biointerfaces, 57, 198-203.

[5] W., Y.Z., W., Y.L., C., G.X., et al. (2000) Preparation and characterization of gelatin gel with a gradient structure. Polymer Internationa, 49, 1600-1603.

[6] Chong, E.J., Phan, T.T., Lim, I.J., et al. (2007) Evaluation of electrospun PCL/gelatin nanofibrous scaffold for wound healing and layered dermal reconstitution. Acta Biomaterialia, 3, 321-330.

[7] Yurong, L., Luke, M.G., Kennedy, J.E., et al. (2009) Thermal behavior and mechanical properties of physically crosslinked PVA/Gelatin hydrogels. Journal of the Mechanical Behavior of Biomedical Materials, doi: 10.1016/j.jmbbm.2009.07.001.

[8] Nagahama, H., Maeda, H., Kashiki, T., et al. (2009) Preparation and characterization of novel chitosan/gelatin membranes using chitosan hydrogel. Carbohydrate Polymers, 76, 255-260.

[9] Li, M., Guo, Y., Wei, Y., et al. (2006) Electrospinning polyaniline-contained gelatin nanofibers for tissue engineering applications. Biomaterials, 27, 2705-2715.

[10] Schiffman, J.D., and Schauer, C.L. (2007) One-Step Electrospinning of Cross-Linked Chitosan Fibers. Bio- 
macromolecules, 8, 2665-2667.

[11] Agarwal, S., Wendorff, J.H. and Greiner, A. (2008) Use of electrospinning technique for biomedical applications. Polymer, 49, 5603-5621.

[12] You, Y.L., Lee, S.J., Min, B.M., et al. (2006) Effect of solution properties on nanofibrous structure of electrospun poly (lactic-co-glycolic acid). Journal of Applied Polymmer Science, 99, 1214-1221.

[13] Mark, M., Naphtali, O.C., Soumen, M., et al. (2005). Saccharide-Peptide Hybrid Copolymers as Biomaterials. Angewandte Chemie International Edition, 44, 65296533.

[14] Buttafoco, L., Kolkman, N.G., Engbers-Buijtenhuijs, P., et al. (2006) Electrospinning of collagen and elastin for tissue engineering applications, Biomaterials, 27, 724-734.

[15] Pham, Q.P., Sharma, U. and Mikos, A.G. (2006) Electrospinning of polymeric nanofibers for tissue engineering applications: A review. Tissue Engenineering, 12, 1197-1211.

[16] Duan, B., Yuan, X., Zhu, Y., et al. (2006) A nanofibrous composite membrane of PLGA-chitosan/PVA prepared by electrospinning. European Polymer Journal, 42, 2013-2022.

[17] Kenawy, E.R., Bowlin, G.L., Mansfield, K., et al. (2002) Release of tetracycline hydrochloride from electrospun poly(ethylene-co-vinylacetate), poly(lactic acid), and a blend Journal of Controlled Release, 81, 57-64.

[18] Shudong, W., Youzhu, Z., Hongwei, W. et al. (2009) Fabrication and Properties of the Electrospun Polylactide/Silk Fibroin-Gelatin Composite Tubular Scaffold. Biomacromolecules, 10, 2240-2244.

[19] Huang, Z.-M., Zhang, Y.Z., Kotaki, M., et al. (2003) Composite Science Technology, 63, 2223-2253.

[20] Zhang, Y.Z., Venugopal, J., Huang, Z.-M., et al. (2006) Crosslinking of the electrospun gelatin nanofibers. Polymer, 47, 2911-2917.

[21] Song, J.-H., Kim, H.-E. and Kim, H.-W. (2008) Production of electrospun gelatin nanofiber by water-based co-solvent approach. Journal of Materials Science: Materials in Medicine, 19, 95-102.
[22] Chen, Z.G., Wang, P.W., Wei, B., et al. (2010) Electrospun collagen-chitosan nanofiber: A biomimetic extracellular matrix for endothelial cell and smooth muscle cell. Acta Biomaterialia, 6, 372-382.

[23] Bigi, A. and Cojazzi, G. (2001) Mechanical and thermal properties of gelatin films at different degrees of glutaraldehyde crosslinking. Biomaterials, 22, 763-768.

[24] Gilberto, G., Junior, E.M., Marcantoö nio, R. A. C., et al. (1999) Biocompatibility studies of anionic collagen membranes with different degree of glutaraldehyde cross-linking. Biomaterials, 20, 27-34.

[25] Sisson, K., C.Z., Farach-Carson, M.C., Chase, D.B. and Rabolt J.F. (2009) Evaluation of cross-linking methods for electrospun gelatin on cell growth and viability. Biomacromolecules, 10, doi: 10.1021/ bm900036s.

[26] Nguyen, T.H. and Lee, B.-T. (2010) Electro-spinning of PLGA/PCL blends for tissue engineering and their biocompatibility. Journal of Materials Science: Materials in Medicine, 21, 1969-1978.

[27] Min, B.-M., You,Y., Kim, J.-M., at el. (2004) Formation of nanostructured poly(lactic-co-glycolic acid)/chitin matrix and its cellular response to normal human keratinocytes and fibroblasts. Carbohydrate Polymers, 57, 285-292.

[28] Martucci, J.F., Ruseckaite, R.A. and V'azquez, A. (2006) Creep of glutaraldehyde-crosslinked gelatin films. Materials Science and Engineering A, 435-436, 681-686.

[29] Talebian, A., Kordestani, S.S., Dadashian F. et al. (2007) World Applied Sciences Journal, 2, 575-581.

[30] ASTM Standard (1991) Standard test method for tensile properties of thin plastic sheeting. In: American Society for Testing and Materials, West Conshohocken, Ed. 194-202.

[31] Russell, A.D., Dancer, B.N. and Power, E.G.M. (1991) Effects of Chemical Agents on Bacterial Sporulation, Germination and Outgrowth. In: Denyer, S.P. and Hugo, W.B. Eds., Mechanisms of Action of Chemical Biocides: Their Study and Exploitation, Blackwell Scientific Publications, Oxford, 23-28. 